\title{
An Unusual Presentation of Cushing's Syndrome
}

Angus Stirling ${ }^{1} \mathrm{MBChB}, \mathrm{MRCP}, \mathrm{PGCert}$

David Carty' MBChB, FRCP, PhD

1 Department of Diabetes, Endocrinology \& Clinical Pharmacology Glasgow Royal Infirmary

84 Castle Street

Glasgow

G4 OSF

Topic: Adrenal and steroids

Introduction: Cushing's syndrome results from chronic exposure to excess glucocorticoids. It is rare, with an incidence of 0.2-5.0 per million people per year, and a prevalence o 39-79 per million people. ' Endogenous causes are divided into adrenocorticotropic hormone (ACTH)-dependent, and $\mathrm{ACTH}$-independent causes. Pituitary corticotroph adenomatous disease (Cushing's disease) is the most common cause of endogenous Cushing's syndrome.

Clinical manifestations include2; proximal myopathies, abnormal fat distribution (particularly in the abdomen, torso and face), disruption to fertility and menstruation, osteoporosis, increased tendency to infection, hypertension, diabetes mellitus, dyslipidaemia, cognitive impairment, psychiatric complications and non-specific symptoms such as fatigue and weight gain.

Hypercortisolism is confirmed with a screening test such as; an overnight dexamethasone suppression test (DST), $24 \mathrm{~h}$ urinary free cortisol, or latenight salivary cortisol. ACTH levels are measured. In ACTH-independent cases an adrenal CT is performed. In ACTH-dependent cases, MRI of pituitary alongside further biochemical assessments such as high dose DST or corticotrophin releasing hormone $(\mathrm{CRH})$ test. Where these tests are concordant, Cushing's disease is confirmed. If not, then bilateral inferior petrosal sinus sampling is performed. Where pituitary disease is excluded, cross-sectional imaging, octreoscan or PET-CT is used to determine an ectopic source of ACTH.

Hypercortisolism can be treated with medical therapy such metyrapone, which selectively inhibits $11 \beta$-hydroxylase, or cabergoline (effective in approximately $30 \%$ of patients) ${ }^{\prime}$. Ketoconazole also reduces endogenous cortisol production, but was withdrawn in Europe due to concerns regarding liver toxicity. Surgical treatment should be considered in adrenal, or pituitary-adenomatous disease or in cases of ectopic ACTH.

Case Presentation: A 50 year old man was admitted to the Acute Assessment Unit at Glasgow Royal Infirmary on 20/8/2017 with left-sided chest pain. A chest radiograph revealed a left-sided hilar mass. Blood pressure was 164/94. A non-fasting glucose sample was $5.1 \mathrm{mmol} / \mathrm{L}$. He was not on treatment for hypertension. Cross-sectional imaging of the thorax demonstrated a $9.2 \mathrm{~cm}$ centrally necrotic mass arising from the anterior mediastinum, abutting the left ventricle, with adjacent ymphadenopathy and multiple bone metastases. A CT-guided biopsy was performed on 04/09/2017, and the pathology was felt to be of an atypical carcinoid tumour.

The patient was referred to clinical oncology. An octreotide scan confirmed a left-sided avid lesion within the thorax. Gut hormone profile was normal. He was commenced on octreotide LAR in November. A further CT in February, following 3 doses of octreotide, demonstrated progression of the primary tumour, lymphadenopathy and skeletal metastases and he was commenced on temozolomide. He was reviewed by cardiothoracic surgery who had concerns regarding his suitability for operative intervention due to his weight - BMl was $42.9 \mathrm{~kg} / \mathrm{m}^{2}$.

The patient was subsequently re-admitted on 19/02/2018 with osmotic symptoms and a laboratory glucose of $21.8 \mathrm{mmol} / \mathrm{L}$ consistent with a diagnosis of diabetes mellitus. He was managed with twice daily insulin and metformin. Despite lacking symptoms of Cushing's syndrome, the cortisol following an overnight dexamethasone suppression test was 1878 $\mathrm{nmol} / \mathrm{L}$ (ref. $<50 \mathrm{nmol} / \mathrm{L}$ ). ACTH was elevated at $258 \mathrm{mU} / \mathrm{L}$ (ref. $<20 \mathrm{mU} / \mathrm{L}$ ) and urinary cortisol was $>2000 \mathrm{nmol} / \mathrm{L}$. The presumed diagnosis was of ectopic ACTH from the known carcinoid tumour.

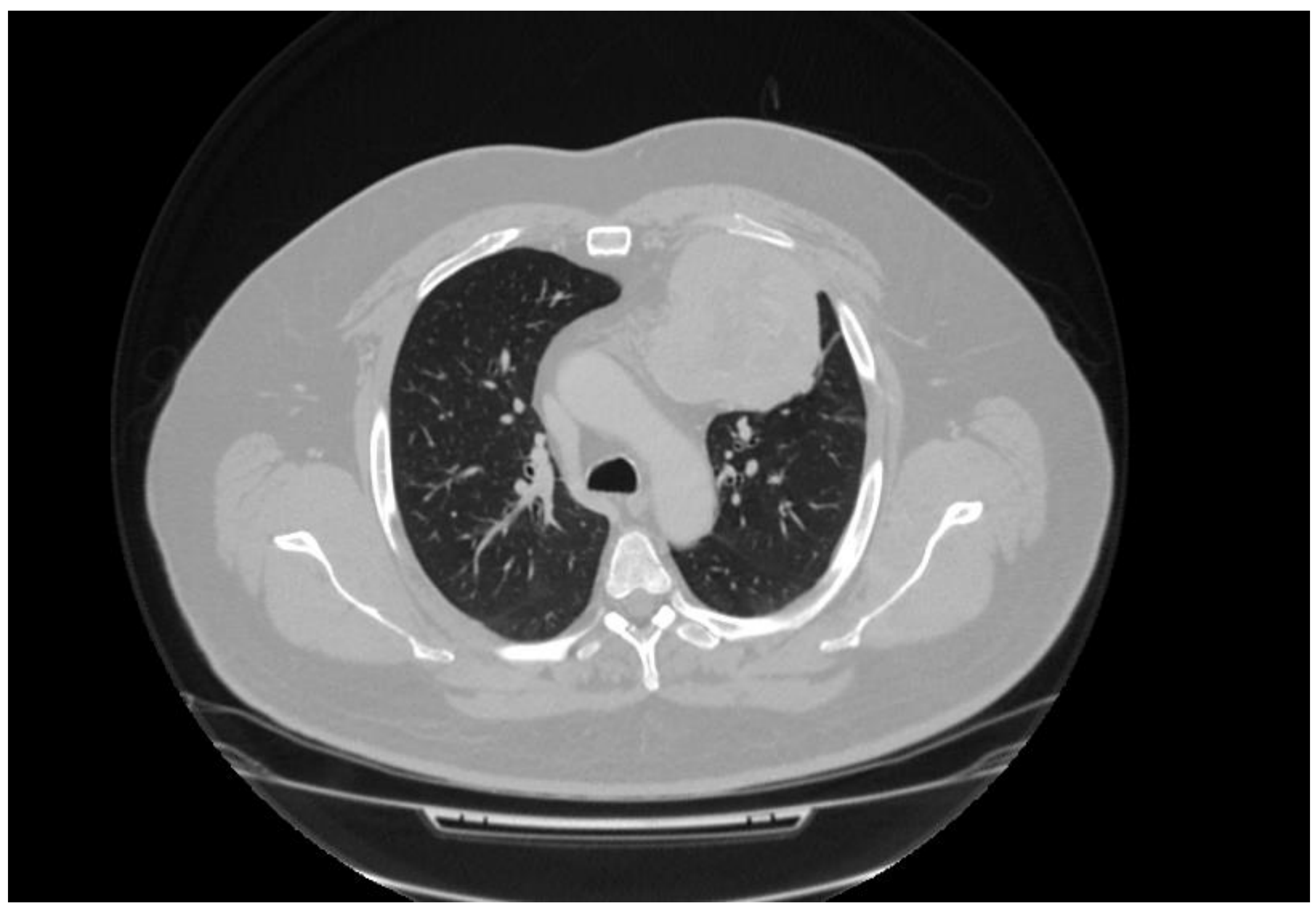

The patient was re-admitted on 14/03/2018 with a hypokalaemia of 2.7 $\mathrm{mmol} / \mathrm{L}$ and was noted to have marked proximal myopathy and peripheral oedema. He was commenced on metyrapone at an initial dose of $250 \mathrm{mg}$, twice daily, which was up-titrated to $1500 \mathrm{mg}$ per day (see figure below). He was commenced on $20 \mathrm{mg}$ in the morning and 10 $\mathrm{mg}$ in the afternoon of hydrocortisone on $28 / 03 / 2018$. His admission was complicated by respiratory sepsis, and he was converted onto intravenous hydrocortisone for several days. Cortisol levels rose and the hydrocortisone was suspended on 02/04/2018 with his levels being monitored regularly. It was resumed on 06/04/2018.

Since discharge he has continued on insulin therapy, metformin, metyrapone and hydrocortisone, alongside octreotide LAR and temozolamide. A CT on 30/07/2018 has shown a slight reduction in the size of the mediastinal mass. BMl had reduced to $38.3 \mathrm{~kg} / \mathrm{m}^{2}$ in September, and $\mathrm{HbA}_{1 \mathrm{c}}$ was $55 \mathrm{mmol} / \mathrm{L}$. He is to be re-discussed with cardiothoracic surgery for consideration of resection of the tumour. If this is felt to be inappropriate, bilateral adrenalectomy will be considered.

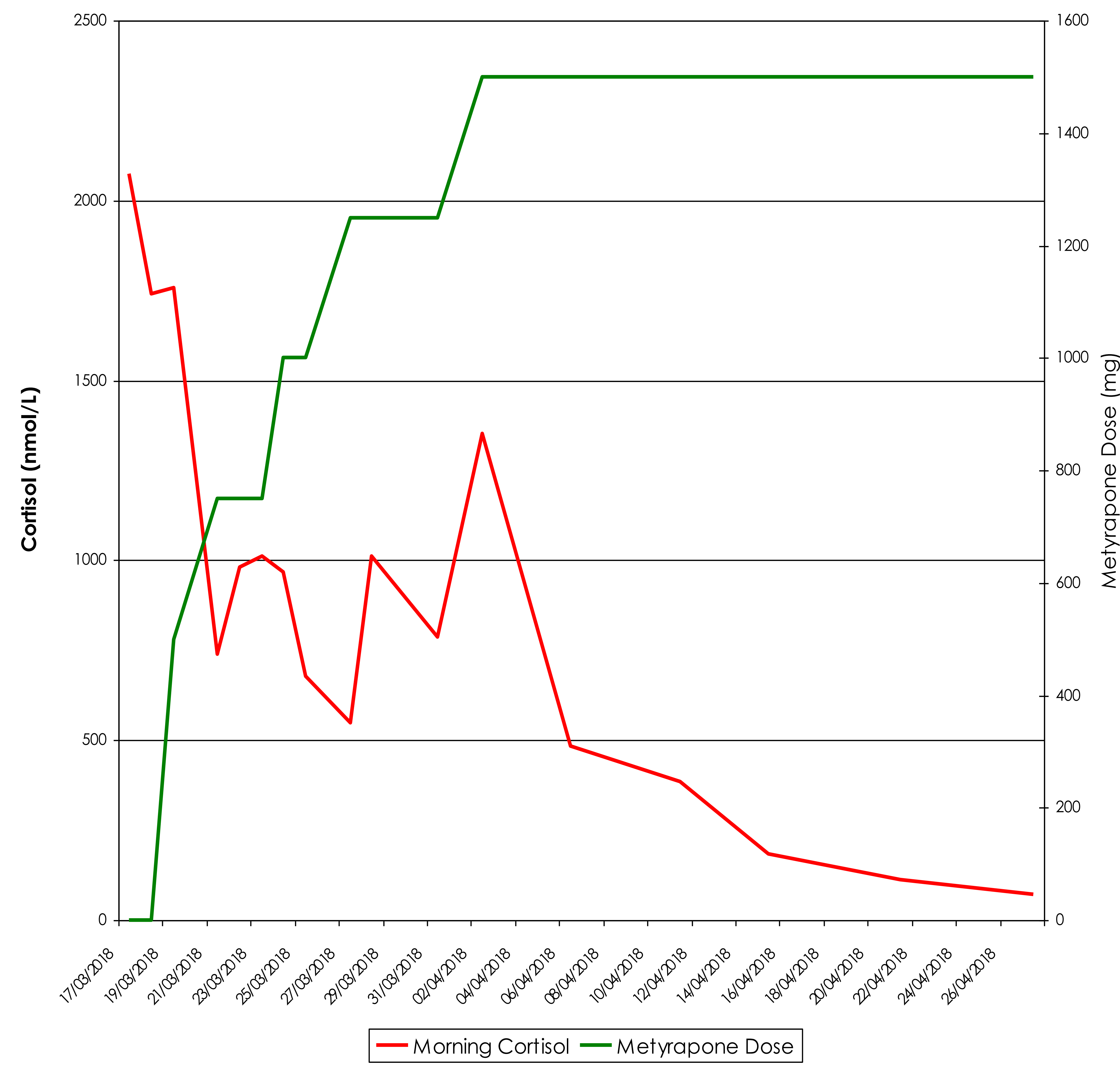

References:

1. Lacroix A, Feelders RA, Stratakis CA, Nieman LK. Cushing's Syndrome. Lancet. 2015;386:913-27 2. Nieman KL. Cushing's Syndrome: Update on signs, symptoms and biochemical screening. Eur J Endocrinol. 2015;173(4):M33-38 